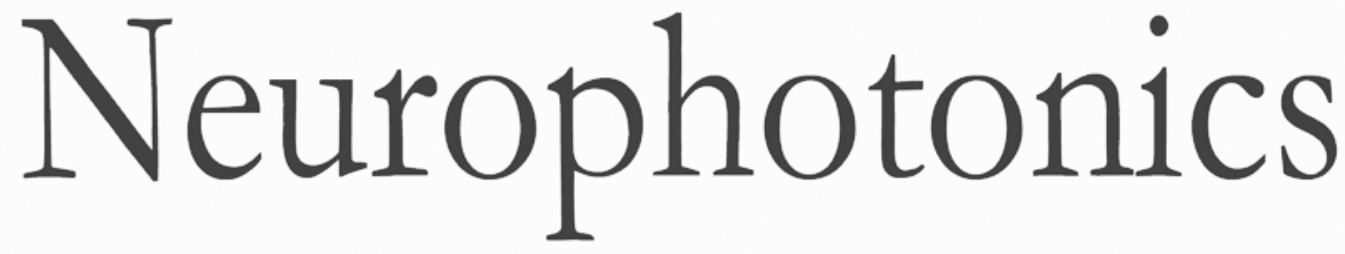

\title{
Microvascular cerebral blood flow fluctuations in association with apneas and hypopneas in acute ischemic stroke
}

Clara Gregori-Pla

Raquel Delgado-Mederos

Gianluca Cotta

Giacomo Giacalone

Federica Maruccia

Stella Avtzi

Luís Prats-Sánchez

Alejandro Martínez-Domeño

Pol Camps-Renom

Joan Martí-Fàbregas

Turgut Durduran

Mercedes Mayos 


\title{
Microvascular cerebral blood flow fluctuations in association with apneas and hypopneas in acute ischemic stroke
}

\author{
Clara Gregori-Pla, a,* Raquel Delgado-Mederos, ${ }^{\mathrm{b}}$ Gianluca Cotta, ${ }^{\mathrm{a}}$ Giacomo Giacalone, ${ }^{\text {a,c }}$ Federica Maruccia, ${ }^{\mathrm{a}, \mathrm{d}}$ \\ Stella Avtzi, ${ }^{a}$ Luís Prats-Sánchez,, Alejandro Martínez-Domeño, ${ }^{b}$ Pol Camps-Renom, ${ }^{b}$ Joan Martí-Fàbregas, \\ Turgut Durduran, ${ }^{\mathrm{a}, \mathrm{e}}$ and Mercedes Mayos ${ }^{\mathrm{f}, \mathrm{g}}$ \\ aCFO-Institut de Ciències Fotòniques, The Barcelona Institute of Science and Technology, Castelldefels (Barcelona), Spain \\ ${ }^{b}$ Hospital de la Santa Creu i Sant Pau, Biomedical Research Institute Sant Pau (IIB Sant Pau), Department of Neurology (Stroke Unit), Barcelona, \\ Spain \\ 'San Raffaele Scientific Institute, Milan, Italy \\ ¿Universitat Autònoma de Barcelona, Neurotraumatology and Neurosurgery Research Unit, Vall d'Hebron University Research Institute, \\ Barcelona, Spain \\ e Institució Catalana de Recerca i Estudis Avançats, Barcelona, Spain \\ fHospital de la Santa Creu i Sant Pau, Sleep Unit, Department of Respiratory Medicine, Barcelona, Spain \\ ${ }^{9} \mathrm{CIBER}$ Enfermedades Respiratorias (CB06/06), Madrid, Spain
}

\begin{abstract}
In a pilot study on acute ischemic stroke (AIS) patients, unexpected periodic fluctuations in microvascular cerebral blood flow (CBF) had been observed. Motivated by the relative lack of information about the impact of the emergence of breathing disorders in association with stroke on cerebral hemodynamics, we hypothesized that these fluctuations are due to apneic and hypopneic events. A total of 28 patients were screened within the first week after stroke with a pulse oximeter. Five (18\%) showed fluctuations of arterial blood oxygen saturation $(\geq 3 \%)$ and were included in the study. Near-infrared diffuse correlation spectroscopy (DCS) was utilized bilaterally to measure the frontal lobe CBF alongside respiratory polygraphy. Biphasic CBF fluctuations were observed with a bilateral increase of $27.1 \% \pm 17.7 \%$ and $29.0 \% \pm 17.4 \%$ for the ipsilesional and contralesional hemispheres, respectively, and a decrease of $-19.3 \% \pm 9.1 \%$ and $-21.0 \% \pm 8.9 \%$ for the ipsilesional and contralesional hemispheres, respectively. The polygraph revealed that, in general, the fluctuations were associated with apneic and hypopneic events. This study motivates us to investigate whether the impact of altered respiratory patterns on cerebral hemodynamics can be detrimental in AIS patients. (๑) The Authors. Published by SPIE under a Creative Commons Attribution 4.0 Unported License. Distribution or reproduction of this work in whole or in part requires full attribution of the original publication, including its DOI. [DOI: 10.1117/1.NPh.6.2.025004]
\end{abstract}

Keywords: diffuse optics; diffuse correlation spectroscopy; stroke; sleep disorders.

Paper 18058RR received Oct. 5, 2018; accepted for publication Apr. 4, 2019; published online Apr. 23, 2019.

\section{Introduction}

Most interventions during the early hours after ischemic stroke onset aim to maximize and stabilize cerebral blood perfusion to diminish the neurological damage and improve the long-term outcome. ${ }^{1}$ Furthermore, these patients could be at risk of possible detrimental cerebral blood flow $(\mathrm{CBF})$ fluctuations in the case, for instance, of undiagnosed breathing disorders (BDs). Disordered breathing includes a range of respiratory disorders, ${ }^{2}$ often during sleep, characterized by repetitive reduction (hypopneas) or cessation (apneas) of airflow.

Different types of BDs with different physiological origins have been reported in association with stroke in $10 \%$ to $70 \%$ of the patients. ${ }^{3-12}$ However, it is not yet clear if the presence of a BD is associated with the long-term outcome. Although some works support the idea that BDs are associated with poor recovery from stroke, ${ }^{10,13-16}$ one study has reported that BDs in stroke patients are associated with early neurological worsening but not with long-term outcome at 6 months. ${ }^{4}$ The reason for the divergence of the results of these studies could be not only the physiological origin of BDs (upper airway obstruction, heart failure, disease, or injury involving the

*Address all correspondence to Clara Gregori-Pla, E-mail: clara.gregori@ alumni.icfo.eu brain $)^{17}$ but also the possible, variable amount of detrimental impact of these events on the cerebral hemodynamics. Thus, the diagnosis, the quantification of these respiratory events, and the characterization of their effect on the cerebral hemodynamics may be relevant for the management of the ischemic stroke patients.

The dynamics of local, microvascular CBF are particularly interesting as, hypothetically, they could reveal that large, repeated changes in the cerebral perfusion could be detrimental when cerebral autoregulation is damaged in the acute stages of stroke. ${ }^{18}$ This is relevant during these acute stages as ischemia in the brain is a potentially reversible process dependent on the restoration of the microvascular $\mathrm{CBF}$ in the penumbra within a time window of cellular viability that varies on duration and severity of CBF cessation. ${ }^{19}$ However, if cerebral autoregulation is damaged, CBF can abruptly increase or decrease without following the needs of the brain and without restoring the needed $\mathrm{CBF}$ in the penumbra. If so, $\mathrm{BD}$ treatment could be used to avoid these unwanted changes. However, there are no established methods to monitor microvascular CBF at the bedside.

In non-stroke subjects, cerebral hemodynamic fluctuations due to apneas or hypopneas have been extensively studied and characterized previously by several groups by measuring the cerebral blood flow velocity $(\mathrm{CBFV})$ in the middle cerebral artery by transcranial Doppler ultrasound (TCD) ${ }^{20-24}$ However, 
macrovascular CBFV is only an indirect measurement of what happens in the distal microcirculation.

Fluctuations of cerebral hemodynamics have previously been observed in acute stroke patients. A study of nine patients with acute stroke ${ }^{25}$ reported arterial desaturations from unknown origin that correlated with cerebral hemodynamics measured with near-infrared diffuse optical spectroscopy (NIRS-DOS), and showed an asymmetry of the changes in the two cerebral hemispheres. Pizza et al. ${ }^{26}$ observed in seven patients with acute/subacute middle cerebral artery stroke that obstructive and central apneas were linked to cerebral oxygenation changes by NIRSDOS. Asymmetrical patterns of cerebral hemodynamic changes were again found with significantly larger changes on the unaffected hemisphere compared to the affected one. However, neither NIRS-DOS nor TCD provide a direct measure of microvascular $\mathrm{CBF}$.

In this pilot study, we have utilized diffuse correlation spectroscopy (DCS), which uses the speckle statistics of near-infrared diffuse light to noninvasively monitor local, microvascular $\mathrm{CBF}^{27-29}$ DCS does not need any exogenous tracers and has been validated against different modalities. ${ }^{29,30}$ In previous studies,${ }^{31}$ we have observed unexpected fluctuations of microvascular $\mathrm{CBF}$, heart rate (HR), arterial oxygen saturation $\left(\mathrm{SpO}_{2}\right)$, and end-tidal carbon dioxide in three (18\%) of the seventeen acute ischemic stroke (AIS) patients during the first week from stroke onset, which has motivated the design of this study to investigate the origin of these fluctuations. Here, we have aimed to test the hypothesis that these cerebral and systemic fluctuations were associated with apneic and hypopneic events after stroke.

\section{Methods}

This study was conducted at the Stroke Unit of Hospital de la Santa Creu i Sant Pau, in Barcelona, Spain, from August 2016 to March 2017. The study protocol was approved by the local ethical committee (EC/15/130). Either the patients or their relatives gave their informed written consent.

The inclusion criteria were being older than 18 years, confirmed ischemic stroke in the anterior circulation, measurement time $\leq 7$ days from the stroke onset and written informed consent. The exclusion criteria were resting $\mathrm{HR}<40$ or $>110$ beats per minute (bmp), $\mathrm{SpO}_{2}<92 \%$, use of oxygen therapy, symptomatic lacunar stroke, previous diagnosis of BDs, previous transient ischemic attack, minor stroke, or intracranial hemorrhage.

The study included two different parts (see Fig. 1, for a flow chart of the procedure). First, patients who fit the clinical inclusion criteria were screened with a pulse oximeter for a minimum of $4 \mathrm{~h}$ between 8 am and $2 \mathrm{pm}$. Afterward, if the pulse oximeter showed periodic decreases of $\geq 3 \%$ in $\mathrm{SpO}_{2}$, the patients were measured in the afternoon with DCS and also with a respiratory polygraphy device simultaneously for $1 \mathrm{~h}$ in the most comfortable head-of-bed position for the patients.

\subsection{Neurological Evaluation}

Baseline examinations included the collection of a summary of the medical history from patient records (demographics and vascular risk factors) and a clinical examination. The stroke severity was assessed with the National Institutes of Health Stroke Scale (NIHSS $^{32}$ on admission and right after the measurement. Indirect ischemic signs were evaluated by the Alberta Stroke Program Early Computed Tomography Score (ASPECTS). ${ }^{33}$ Long-term outcome was evaluated at 3 months by means of the modified Rankin Scale (mRS) ${ }^{34}$ where a score of $>2$ was considered indicative of an unfavorable outcome.

The extent and location of the ischemic area along with the site of arterial occlusion were evaluated on admission by a cranial computed tomography scan and vascular imaging either by angio-computed tomography or by TCD. If patients were eligible, reperfusion therapy (thrombolysis or thrombectomy) was applied. ${ }^{35}$ Recanalization (either by spontaneous or by revascularization treatment) was assessed with TCD within 24 to $48 \mathrm{~h}$.

The neurological scales were scored by a neurologist or a senior neurology resident blinded to the optical information.

\subsection{Optical Methods and Instrumentation}

We have used a custom-built DCS, ${ }^{27}$ which was previously described in other protocols within the same collaboration. ${ }^{36-39}$ Two optical probes were placed on the forehead bilaterally on both hemispheres as lateral as possible, avoiding the sinuses. These probes consisted of a bundle of four detector fiber set at $2.5 \mathrm{~cm}$ from a source fiber. We have measured the blood flow index (BFI) continuously with a 2.5-s temporal resolution. The relative $\mathrm{CBF}$ changes were obtained by normalizing the calculated continuous BFI with the mean BFI of the first 5 min (baseline) of the measurement. Also, the CBF changes per event were calculated, as explained in Sec. 2.3.

\subsubsection{Pulse oximetry and respiratory polygraphy}

A standard pulse oximeter (CMS-50D Plus, CONTEC Medical Systems CO, Qinhuangdao, China) was used for the screening of the stroke patients. If these patients were eligible, then they

\section{Part 2}

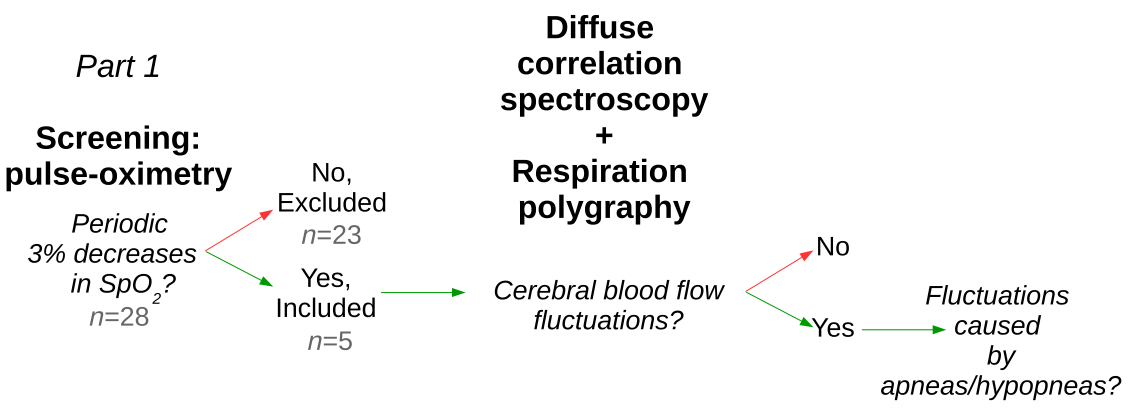

Fig. 1 Methodology of the two parts of the study. The subjects included in each part are depicted in gray. $\mathrm{SpO}_{2}$, arterial oxygen saturation. 
were enrolled for the full study which, in addition to DCS, included a respiratory polygraphy monitoring (Embletta MPR PG, Natus Medical, Middleton, Wisconsin).

The respiratory polygraphy included the recording of the oronasal flow (by a thermistor and a nasal cannula), the thoracic and the abdominal movements (by a respiratory inductance plethysmography band), the $\mathrm{SpO}_{2}$ and the $\mathrm{HR}$ (both by pulse oximetry), among others. The respiratory polygraphy data were further postprocessed by a pulmonologist to determine the following variables: the start and end time points of each event, the event type (obstructive apnea, hypopnea, mixed apnea, or central apnea), the $3 \%$ oxygen desaturation index (ODI3), and the number of apneic and hypopneic events per hour of study $(\mathrm{AHI}) .^{40}$

\subsection{Statistical Analysis}

To calculate the percentage of $\mathrm{CBF}$ change $(\Delta \mathrm{rCBF})$ due to each apneic or hypopneic event, we have estimated a basal BFI $\left(\mathrm{BFI}_{\mathrm{bl}}\right)$ as the average of the cerebral BFI from $30 \mathrm{~s}$ before the apnea/hypopnea start up to $30 \mathrm{~s}$ after the event end. This was considered to correct for slight changes in the probe position during the measurement. Afterward, the $\mathrm{CBF}$ changes were calculated as $\Delta r \mathrm{CBF}=\left(\frac{\mathrm{BFI}}{\mathrm{BFI}_{\mathrm{bl}}}-1\right) \times 100$. Similarly, $\Delta \mathrm{HR}$ was defined as $\Delta \mathrm{HR}=\mathrm{HR}-\mathrm{HR}_{\mathrm{bl}}$ and $\Delta \mathrm{SpO}_{2}$ was defined as $\Delta \mathrm{SpO}_{2}=\mathrm{SpO}_{2}-\mathrm{SpO}_{2 \mathrm{bl}}$. In all cases, the subscript ${ }_{b l}$ indicates an average over the same period.

Afterward, the events were parametrized for the characterization of the induced $\Delta \mathrm{rCBF}, \Delta \mathrm{HR}$, or $\Delta \mathrm{SpO}_{2}$ for each event. The goal was to study if individual apneic or hypopneic events were associated with significant increases or decreases in these variables as it was hypothesized. This step consisted in considering each event as a function dependent on time $[\Delta \mathrm{rCBF}(\mathrm{t})$, $\Delta \mathrm{HR}(\mathrm{t})$, and $\left.\Delta \mathrm{SpO}_{2}(\mathrm{t})\right]$, and then, the extrema of these functions along a specific time interval were calculated. The reference time (time zero) was considered as the end of each event. The time windows to find these extrema were considered from -5 to $15 \mathrm{~s}$ for the first extremum on $\triangle \mathrm{rCBF}$ (see Fig. 2, as an example), from 0 to $15 \mathrm{~s}$ for the $\Delta \mathrm{HR}$, and from 5 to $35 \mathrm{~s}$ for the $\Delta \mathrm{SpO}_{2}$. These time windows were chosen by visual observation of all the apneas and hypopneas plotted together from -30 to $60 \mathrm{~s}$ and according to the literature. ${ }^{20,41}$ These analysis methods have previously been verified in nocturnal measurements of chronic obstructive sleep apnea patients. ${ }^{42}$

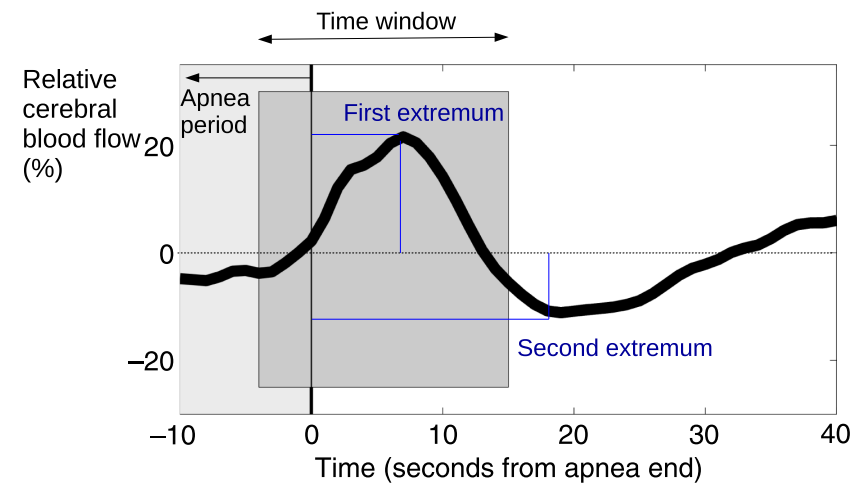

Fig. 2 Cerebral blood flow characterization of individual sleep events, where the apnea end is considered as a pivot point. The light gray region indicates the sleep event. The dark gray region indicates the time window used for finding the first extremum value. The first and second extrema are labeled in blue.
Averages and standard deviations have been performed considering all apneic and hypopneic events for each patient and also for the population. The Wilcoxon signed-rank test was used to assess the difference between ipsilesional and contralesional $\triangle \mathrm{rCBF}$ responses. The same test was used to assess if the apneic/hypopneic response of different variables was significantly different from zero. All analyses were performed with MATLAB (Mathworks, Natick, Massachusetts). A $p$-value $<0.05$ was considered to be statistically significant.

\section{Results}

During the 8-month period, we have screened 28 ischemic stroke patients with pulse oximetry, of whom $5(n=5,18 \%)$ presented fluctuations $(\geq 3 \%)$ in $\mathrm{SpO}_{2}$ and were then included in the second part of the study. Table 1 shows the clinical characteristics of these five patients. Four were male, had an age of $81 \pm 6$ years (mean \pm standard deviation), an admission NIHSS of $8(2,16)$ [median (first quartile, third quartile)], and a time from stroke onset to measurement of $4(3,5)$ days.

Table 2 shows the results of the DCS and the respiratory polygraphy measurements. In all five patients, the respiratory polygraphy confirmed the presence of altered breathing patterns; two presented periodic breathing [cases 1 and 3; clusters of breaths separated by intervals of apnea (no breathing) or hypopnea], two presented a predominance of obstructive and hypopneic events (cases 2 and 4; the airflow ceased or partially ceased due to upper airway obstruction), and one presented central apneas (case 5; the airflow ceased or partially ceased due to diminished or absent effort to breath).

Figure 3 shows a representative trace (from case 4 ) of $10 \mathrm{~min}$ of continuous oronasal flow, abdominal and thoracic movements, $\mathrm{HR}, \mathrm{SpO}_{2}$, and $\mathrm{CBF}$ changes. This specific interval shows two types of events with good signal-to-noise ratio in the optical data and a "close-up" revealing details. Both apneic (four obstructive apneas, green shading) and hypopneic (three, yellow shading) events are visible and the simultaneous monitoring shows time correlated variation of microvascular CBF, $\mathrm{HR}$, and respiratory variables. From the polygraphy recording, oronasal flow appears to be reduced during the hypopneas and ceases during the obstructive apneas, whereas abdominal and thoracic movements are reduced during these events. HR does not follow a general pattern for this trace. $\mathrm{SpO}_{2}$ shows a drop with a delay relative to the apneic/hypopneic event. In this time period with frequent events, as commonly observed, the $\mathrm{SpO}_{2}$ drop of the previous event is overlapped with the next event. It can also be observed that the $\mathrm{CBF}$ reaches its maximum at the end of an event. Finally, for this specific interval, periodic breathing is diagnosed but the rest of the acquisition is dominated by obstructive apneic events, as shown in Table 2 .

On the contrary, Fig. 4 shows 10 min of similar traces where neither fluctuations nor apnea/hypopnea events are found. This patient (case 3) is the only one with no apneic/hypopneic events during those $10 \mathrm{~min}$.

For completeness, the full data set for the five patients can be found in Sec. 5 Appendix.

From the five patients, $239(n=239)$ apneic/hypopneic events were analyzed. In all patients, the CBF changes were biphasic. In other words, significant bilateral CBF increases of $27.1 \% \pm 17.7 \%(p<0.001)$ for the ipsilesional and $29.0 \% \pm$ $17.4 \%(p<0.001)$ for the contralesional hemispheres were followed by decreases of $-19.3 \% \pm 9.1 \%(p<0.001)$ for the ipsilesional and $-21.0 \% \pm 8.9 \%(p<0.001)$ for the contralesional 
Table 1 Demographics and prestroke clinical characteristics (top) and poststroke clinical characteristics (bottom) of the five ischemic stroke patients.

\begin{tabular}{|c|c|c|c|c|c|}
\hline Case & 1 & 2 & 3 & 4 & 5 \\
\hline Age $(y)$, gender $(M / F)$ & $83, \mathrm{~F}$ & $73, \mathrm{M}$ & $82, \mathrm{M}$ & $89, \mathrm{M}$ & $76, \mathrm{M}$ \\
\hline Smoking & Former & Former & Former & Former & Former \\
\hline Arterial hypertension & Yes & Yes & No & No & No \\
\hline Dyslipidemia & Yes & Yes & No & No & No \\
\hline Diabetes & No & No & Yes & No & Yes \\
\hline Ipsilesional ICA stenosis $>70 \%$ & Yes & No & No & - & No \\
\hline Coronary disease & No & No & Yes & No & Yes \\
\hline Atrial fibrillation & No & No & No & No & No \\
\hline Left ventricular ejection fraction (\%) & $>50$ & 40 to 50 & 40 & - & 25 \\
\hline Arterial occlusion & Left MCA & Left MCA & Right ACA & Left MCA & Right MCA \\
\hline NIHSS admission & 7 & 7 & 3 & 20 & 21 \\
\hline \multicolumn{6}{|l|}{ NIHSS after the } \\
\hline measurement & 2 & 1 & 8 & 16 & 21 \\
\hline Reperfusion therapy & Yes & Yes & No & No & No \\
\hline Recanalization & Yes & No & Yes & No & No \\
\hline Infarct location & Insular and frontal & Insular and frontal & Frontal & $\begin{array}{l}\text { Insular, temporal, } \\
\text { and basal ganglia }\end{array}$ & $\begin{array}{l}\text { Insular, temporal, } \\
\text { and parietal }\end{array}$ \\
\hline ASPECTS & 8 & 8 & 10 & 6 & 6 \\
\hline Days from stroke to measurement & 7 & 4 & 3 & 5 & 0 \\
\hline mRS 3 months & 2 & 1 & 4 & 6 & 6 \\
\hline
\end{tabular}

Note: M, male; F, female; ICA, internal carotid artery; MCA, middle cerebral artery; ACA, anterior cerebral artery; NIHSS, National Institutes of Health Stroke Scale; ASPECTS, Alberta Stroke Program Early Computed Tomography Score; and mRS, modified Rankin Scale.

hemispheres were found due to each apneic/hypopneic event. There was no interhemispheric difference between the maximum increases $(p=0.189)$ or the maximum decreases $(p=0.053)$. However, this result suggested a trend toward a potential difference between hemispheres, as it has been previously found by NIRS-DOS, ${ }^{25,26}$ as mentioned in Sec. 1 . Also, as expected, $\mathrm{HR}$ increased $(p<0.001)$ by $6.5 \pm 6.8 \mathrm{bpm}$ and $\mathrm{SpO}_{2}$ decreased $(p<0.001)$ by $-4.9 \% \pm 16.8 \%$.

The two patients (cases 4 and 5) with the highest AHI, one with predominance of an obstructive apnea pattern and the other with predominance of a central apnea pattern, had the two highest NIHSS both on admission and after the measurement and the lowest ASPECTS. Unfortunately, both had a mRS of 6, i.e., they had died during the 3 months after stroke onset. Of these two patients, the one with the highest AHI (case 5) also presented the lowest left ventricular ejection fraction. However, neither of these two patients presented the highest physiological or cerebral hemodynamic changes in response to the respiratory events. One of the characteristics of the remaining three patients (cases 1, 2, and 3) with lower stroke severity was that they presented mainly hypopneic events, in comparison to the obstructive and central apneas found in the two most severe patients (cases 4 and 5). We note that, given the small population, these were just anecdotal statements and should not be considered generalizable.

\section{Discussion}

In this study, we have followed up on our previous observation of unexpected bilateral fluctuations in microvascular CBF in patients with anterior, cerebral ischemic stroke that were observed in different protocols with DCS measurements. ${ }^{31}$ We have hypothesized that these fluctuations were due to BDs, in particular, due to apneic and hypopneic events. Therefore, a protocol based on prescreening for these events with a pulse oximeter was introduced to enroll patients without a previous diagnosis of BDs for follow-up study combining respiratory polygraphy and DCS. A careful evaluation was carried out to identify the origin of these fluctuations and to characterize their details by combining this recording of different respiratory variables, $\mathrm{SpO}_{2}$, and $\mathrm{HR}$. Apneic and hypopneic events were determined to be associated with the effect of cerebral hemodynamics and this effect was characterized. 
Table 2 Polygraphic characteristics (top) and maximum $\mathrm{CBF}, \mathrm{HR}$ and $\mathrm{SpO}_{2}$ changes due to each apneic/hypopneic event (bottom) of each patient.

\begin{tabular}{|c|c|c|c|c|c|}
\hline Case & 1 & 2 & 3 & 4 & 5 \\
\hline $\mathrm{AHI}(n /$ hour $)$ & 45.6 & 13 & 24.8 & 45.8 & 63.8 \\
\hline ODI3 (\%) & 46.3 & 15.7 & 17.4 & 48.1 & 10 \\
\hline Obstructive apneas, $n$ & 0 & 0 & 1 & 31 & 1 \\
\hline Central apneas, $n$ & 1 & 0 & 2 & 0 & 82 \\
\hline Mixed apneas, $n$ & 0 & 0 & 1 & 0 & 0 \\
\hline Hypopneas, $n$ & 37 & 13 & 20 & 30 & 0 \\
\hline Event duration (s) & $21 \pm 7$ & $24 \pm 4$ & $27 \pm 8$ & $30 \pm 11$ & $19 \pm 5$ \\
\hline BD & Periodic respiration & Hypopneas & Periodic respiration & Obstructive events & Central apneas \\
\hline Max. increase-induced $\Delta \mathrm{rCBF}_{\mathrm{ipsi}}(\%)$ & $30.0 \pm 12.7$ & $37.4 \pm 13.0$ & $22.4 \pm 8.9$ & $31.0 \pm 10.1$ & $21.9 \pm 23.9$ \\
\hline Max. increase-induced $\Delta \mathrm{rCBF}_{\text {contra }}(\%)$ & $32.9 \pm 21.4$ & $27.6 \pm 11.0$ & $29.7 \pm 15$ & $36.1 \pm 11.2$ & $21.7 \pm 18.3$ \\
\hline Max. decrease-induced $\Delta \mathrm{rCBF}_{\mathrm{ipsi}}(\%)$ & $-19.2 \pm 7.0$ & $-33.3 \pm 12.1$ & $-16.1 \pm 8.2$ & $-24.2 \pm 6.6$ & $-13.6 \pm 5.8$ \\
\hline Max. decrease-induced $\Delta \mathrm{rCBF}_{\text {contra }}(\%)$ & $-17.8 \pm 7.4$ & $-25.4 \pm 11.1$ & $-19.9 \pm 8.1$ & $-28.0 \pm 8.1$ & $-16.3 \pm 5.3$ \\
\hline Max. increase-induced $\Delta \mathrm{HR}(\mathrm{bpm})$ & $2.8 \pm 1.6$ & $14.8 \pm 8.0$ & $16.0 \pm 6.8$ & $10.6 \pm 4.3$ & $1.1 \pm 0.7$ \\
\hline Max. decrease-induced $\Delta \mathrm{SpO}_{2}(\%)$ & $-2.2 \pm 1.0$ & $-1.6 \pm 0.7$ & $-1.5 \pm 9.3$ & $-9.9 \pm 24.6$ & $-0.9 \pm 0.8$ \\
\hline
\end{tabular}

Note: $\mathrm{AHI}$, apnea-hypopnea index; ODI3, $3 \%$ oxygen desaturation index; $\Delta \mathrm{rCBF}$, relative cerebral blood flow changes; $\Delta \mathrm{HR}, \mathrm{HR}_{\mathrm{c}}$ changes; $\Delta \mathrm{SpO} \mathrm{O}_{2}$, arterial oxygenation saturation changes; Max., maximum; ipsi., ipsilesional hemisphere; contra., contralesional hemisphere; and $n$, number of events detected; mean \pm standard deviation.

As observed by the full data set in the Sec. 5 Appendix, the precise nature of the cause of the microvascular CBF changes is not absolutely clear from this study, i.e., some CBF fluctuations lead the apnea/hypopnea, whereas others follow it. This may be partially due to errors in the precise synchronization of the measurements but it may also have further implications indicating secondary events that may have caused respiratory effects, such as cortical spreading depression ${ }^{43-45}$ in the brain stem. ${ }^{46,47}$ However, our study did not gather definitive evidence (e.g., recordings at multiple locations to demonstrate any spreading activity and/or recordings of the electrical activity) to accept or refute this possibility. These results have encouraged us to look further and deeper into this issue in future studies by combining multimodal, precisely synchronized diffuse optical recordings with polygraphy, capnography, and electroencephalography, and a larger head coverage with multiple source and detector pairs.

By the polygraphy, $\mathrm{HR}$ and $\mathrm{SpO}_{2}$ changes were found during these events. These $\mathrm{HR}$ and $\mathrm{SpO}_{2}$ changes followed the apneic/ hypopneic dynamics expected by the literature in patients with $\mathrm{BDs},{ }^{20,24,41,48,49}$ showing an increase during the event or at the end of it for the HR, and a decrease after the end of the event for the $\mathrm{SpO}_{2}$. No periodicity in the respiratory changes was found, as shown in the full data set in Sec. 5 Appendix, as the periodicity depended on the pathogenic mechanism, on the sleep stage, and on the position, among others. ${ }^{50}$

In non-stroke patients with BDs, several studies have reported CBFV changes measured by TCD as showing an increase close to the end of the apnea. ${ }^{20,23,24}$ Our findings are similar; we have observed a CBF increase of $27.1 \% \pm 17.7 \%$ and $29.0 \% \pm 17.4 \%$ for the ipsilesional and contralesional hemispheres, respectively, and a decrease of $-19.3 \% \pm 9.1 \%$ and $-21.0 \% \pm 8.9 \%$ for the ipsilesional and contralesional hemispheres, respectively, close to the end of the apneic/hypopneic events. This increase is similar to the CBF increase of $30 \% \pm 17 \%$ and the decrease of $-20 \% \pm 12 \%$ described previously by us using DCS in obstructive sleep apneic events. ${ }^{42}$ It is also consistent with the CBFV of $22 \%$ to $42 \%$ increase found by Alex et al. ${ }^{23}$ and also to the $14.6 \% \pm 14 \%$ increase right after the apnea end by Bålfors and Franklin. ${ }^{20}$ However, other authors have found larger CBFV increases. ${ }^{21,24}$

These results show that there is a decrease in cerebral perfusion due to an apneic or hypopneic event. If these intermittent decreases lead to ischemia, they can cause hypoxic/ischemic brain injury, especially if cerebrovascular reactivity and regulation are impaired, which is often the case in ischemic stroke patients. ${ }^{51,52}$ Consequently, this could lead to a worsened prognosis, which may call for interventions such as continuous positive airway pressure (CPAP) therapy. We note that, to date, no direct link among a degree of microvascular CBF changes, the longterm outcome, and the use of CPAP therapy has been studied.

Several works have studied the general effect of CPAP therapy, mainly in obstructive disorders or adaptive servo-ventilation (ASV) treatment for central disorders to avoid apneas and hypopneas. ${ }^{53-55}$ In stroke patients, whereas some authors support the benefits of the CPAP treatment on BDs, ${ }^{15,56-61}$ others claim that these benefits are not clear ${ }^{62,63}$ or that there is no benefit at all. ${ }^{64}$ Nevertheless, as mentioned earlier, BDs diagnosis and CPAP or ASV treatment are not the common practice in the stroke units. 

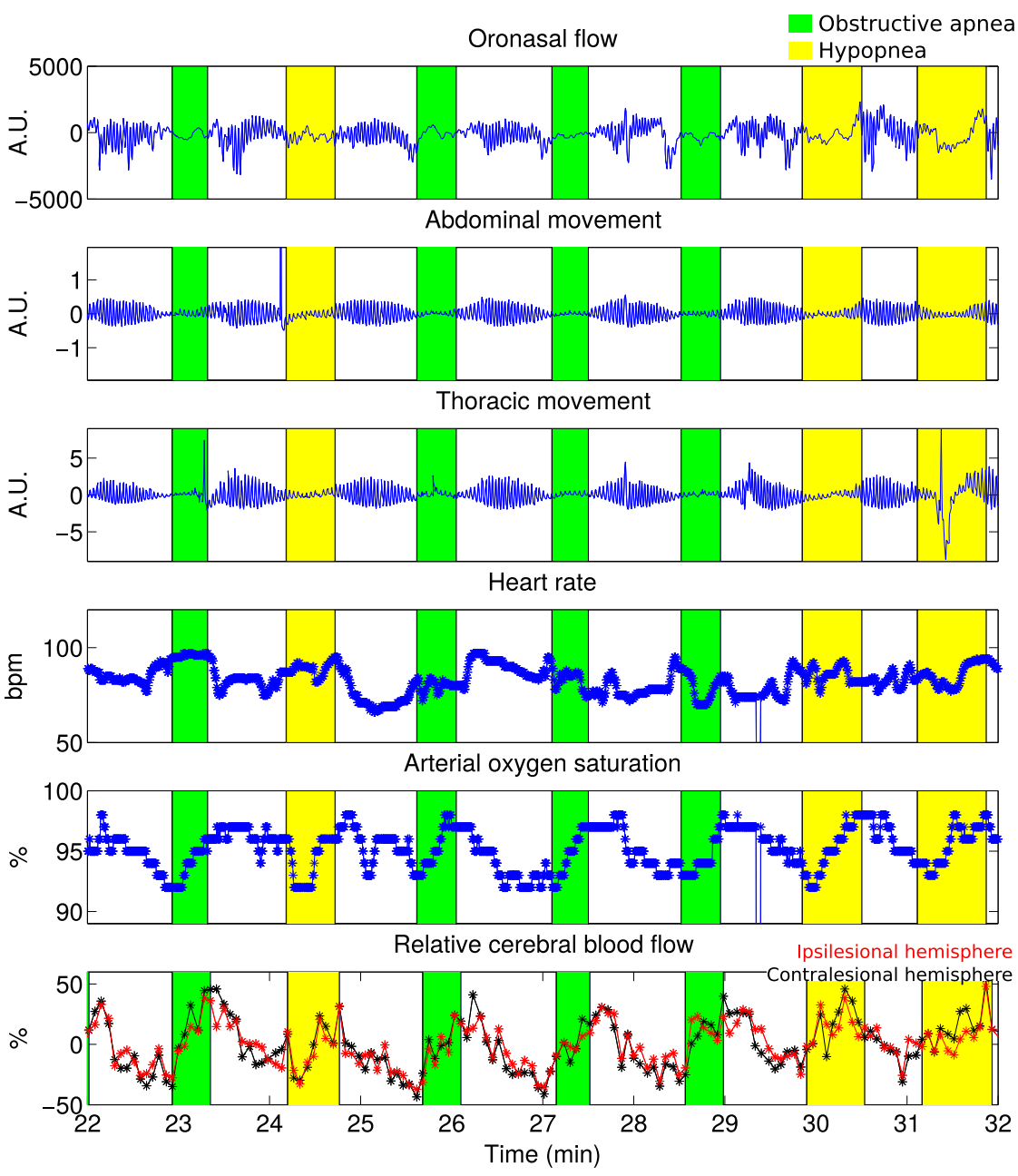

Fig. 3 A 10-min trace of systemic fluctuations of physiological variables alongside the fluctuations of CBF. Red and black CBF traces correspond to the ipsilesional and contralesional hemispheres, respectively. Hypopneas (yellow) and obstructive apneas (green) are shown from the measurements of an 89year-old male with a left middle cerebral artery ischemic stroke (case 4). The patient presented a left middle cerebral artery stroke with insular, temporal, and basal ganglia ischemic areas. The NIHSS score was 16 when measured. The patient died after $24 \mathrm{~h}$ of the measurement.

Future work with a larger sample size to investigate if the CPAP or ASV could be effective in those patients who show the presence and/or large fluctuations in CBF (e.g., above a threshold) in relation to apneic/hypopneic events is warranted. In addition, it would be of interest to study if all ischemic stroke patients with apneic/hypopneic events show similar CBF fluctuations, or if there is an interindividual variability in the response to apneas or hypopneas, and, consequently, the response to treatment may be different.

In our cohort, the two patients (cases 4 and 5) with the highest AHI were those with the worse stroke severity (higher NIHSS score) at the moment of the measurement. This has been previously reported in patients with stroke showing central BDs. ${ }^{13}$ Similarly, the ASPECTS score of these two patients were the lowest of the group, which was compatible with the idea that large acute cerebral hemispheric lesions were associated to BDs. ${ }^{7,65}$ One of these two severe patients (case 5; case 4 had no information available) presented heart failure [low left ventricular ejection fraction $(<40 \%)$ ] and mainly central events, as it has been found in stroke patients in association with central BDs. ${ }^{6}$ The BD present in this patient could be related to it.
Insular and frontal ischemic regions were present in our cohort and, interestingly, these regions have already been associated to BDs of central origin. ${ }^{13,65,66}$ However, other works have supported the idea of the lack of an association between the presence of BDs of central origin and the location of stroke. ${ }^{5,9,11}$ Nevertheless, more patients are needed to clearly identify clinical associations.

Our proof-of-concept study has some limitations, including the small number of patients included in the final analysis, the different types of possible BDs with different physiological origins, and the short recording time of DCS simultaneously with respiratory polygraphy. Future studies will add more subjects and will measure these subjects during several hours continuously in order to better identify the type of $\mathrm{BD}$, and moreover, to link it to the degree of the cerebral hemodynamic responses, among other previously mentioned goals.

Another limitation is that only one measurement per patient has been performed for this study, so we could not track the start and cessation of the fluctuations together with the clinical stroke evolution. Anecdotally, in our previous protocols, ${ }^{31,67}$ the physiological fluctuations observed have 

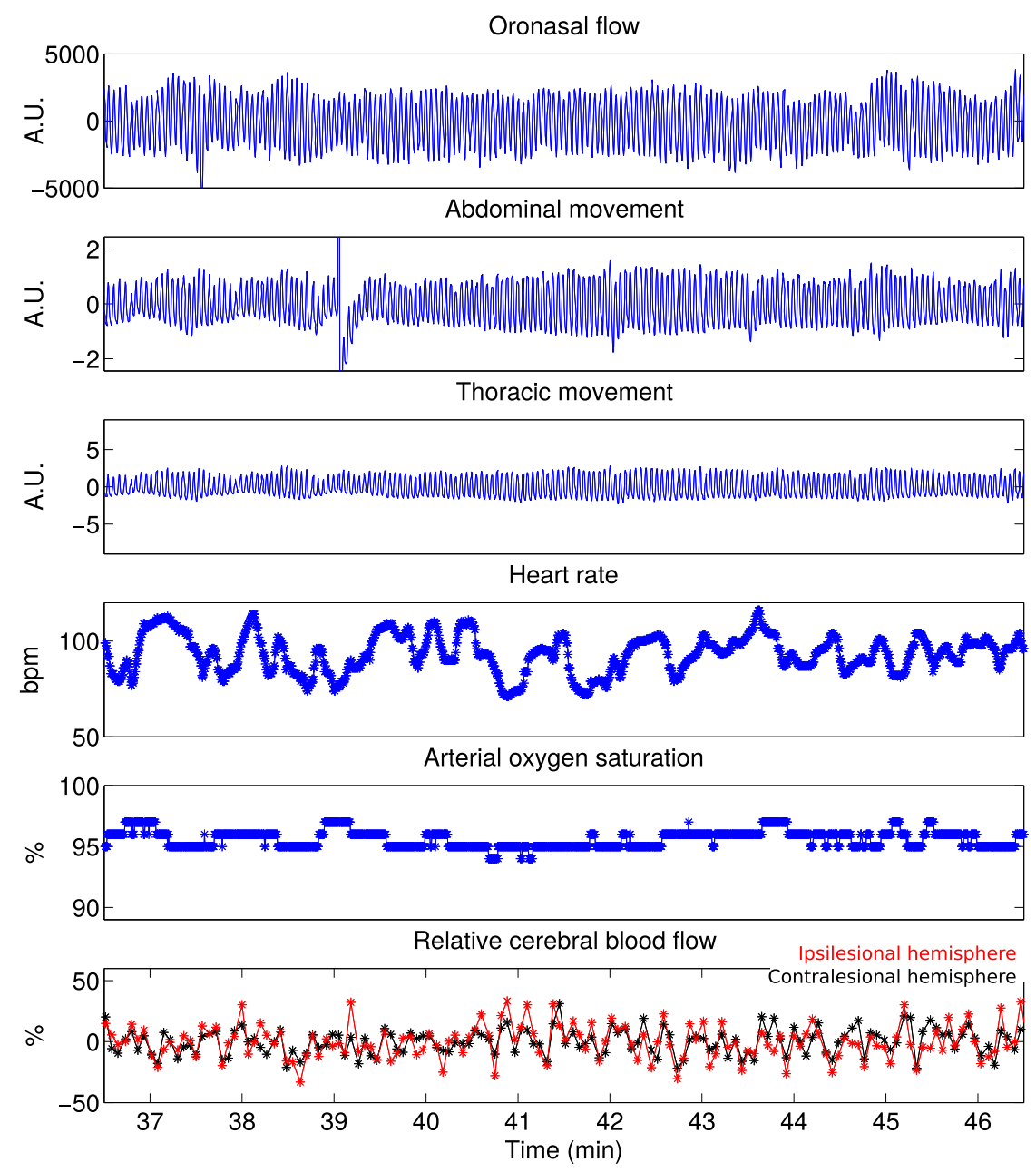

Fig. 4 A 10-min trace with no typical fluctuations of cerebral blood and no apneas or hypopneas. Red and black CBF traces correspond to the ipsilesional and contralesional hemispheres, respectively. Measurements of an 82-year-old male with a right anterior cerebral artery ischemic stroke (case 3) are provided. The patient had a blockage of the right anterior cerebral artery with a frontal ischemic area. The NIHSS score was 8 when measured.

prevailed in the different measurements performed in each patient during the first week after stroke. Accordingly, it is expected that BDs could be present during the first week after stroke in the case of patients with BDs of central origin, as BDs have been found to resolve after several weeks. ${ }^{66}$ Future studies should include daily measurements to track when these fluctuations cease and to relate it to clinical parameters.

Owing to the small number of subjects considered for this analysis, we have avoided a group analysis. Two important parameters to understand CBF changes are the blood pressure and the end-tidal carbon dioxide; future studies should include their simultaneous measurement with DCS. Different studies have suggested these two parameters as different primary drivers to the CBFV changes during apneas. Przybylowski et al. ${ }^{68}$ suggested that carbon dioxide is the primary regulator, and not the blood pressure, of the rise in CBFV (measured by TCD) during apnea and in the reduction of CBFV in the following apnea. On the contrary, Yaggi and Mohsenin ${ }^{69}$ suggested that if autoregulation is impaired or the changes in blood pressure are sufficiently rapid that an intact autoregulation does not have time to adapt, then the changes in TCD velocity could be related to the changes in blood pressure. However, these studies only focus on the macrocirculation level; on the microcirculation level, these parameters may have a different influence on microvascular CBF. Moreover, hypoxia has been found during apneic events, ${ }^{70,71}$ which could also contribute to the local microvascular change.

Finally, diffuse optical spectroscopy techniques not only provide information from the brain but also contain scalp and skull contributions. ${ }^{29,72}$ In this work, instead of including a small source-detector separation $(1 \mathrm{~cm})$ to study the superficial effects, we have kept all detectors for large separations to focus on improving the signal-to-noise ratio, as a source-detector separation of $2.5 \mathrm{~cm}$ has been found to be a good compromise. ${ }^{29,30,73}$ Future implementations could utilize more sourcedetector pairs as well as a pressure modulation protocol to minimize this effect. ${ }^{74}$

In conclusion, DCS has successfully revealed bilateral fluctuations in $\mathrm{CBF}$ of ischemic stroke patients with undiagnosed BDs, in accordance with systemic fluctuations of physiological variables. These cerebral hemodynamic fluctuations are related to apneic and hypopneic events in the five patients tested. Further studies are needed to study the possible detrimental 
impact of the magnitude and presence of these cerebral hemodynamic fluctuations, especially in the early hours after stroke where the interventions are more relevant to the outcome, for the recovery of stroke patients.

\section{Appendix}

The full data set for the five patients measured is shown in Figs. 5-9.
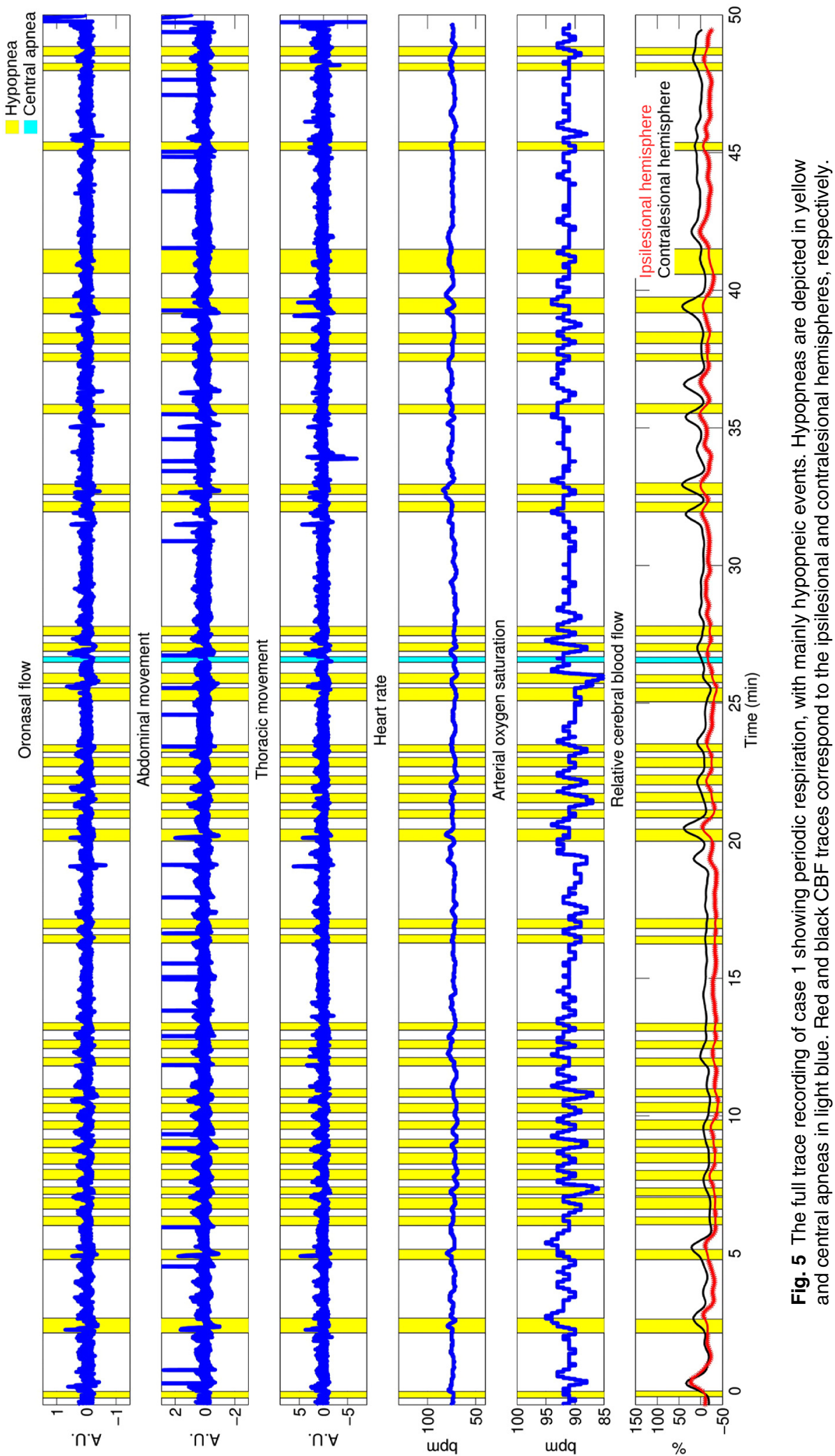

Neurophotonics

025004-8

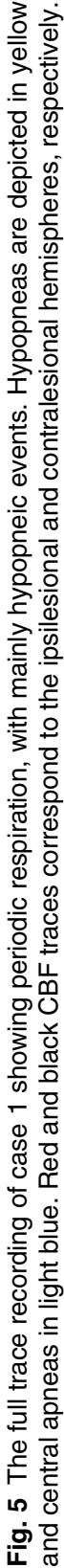

Apr-Jun 2019 • Vol. 6(2) 


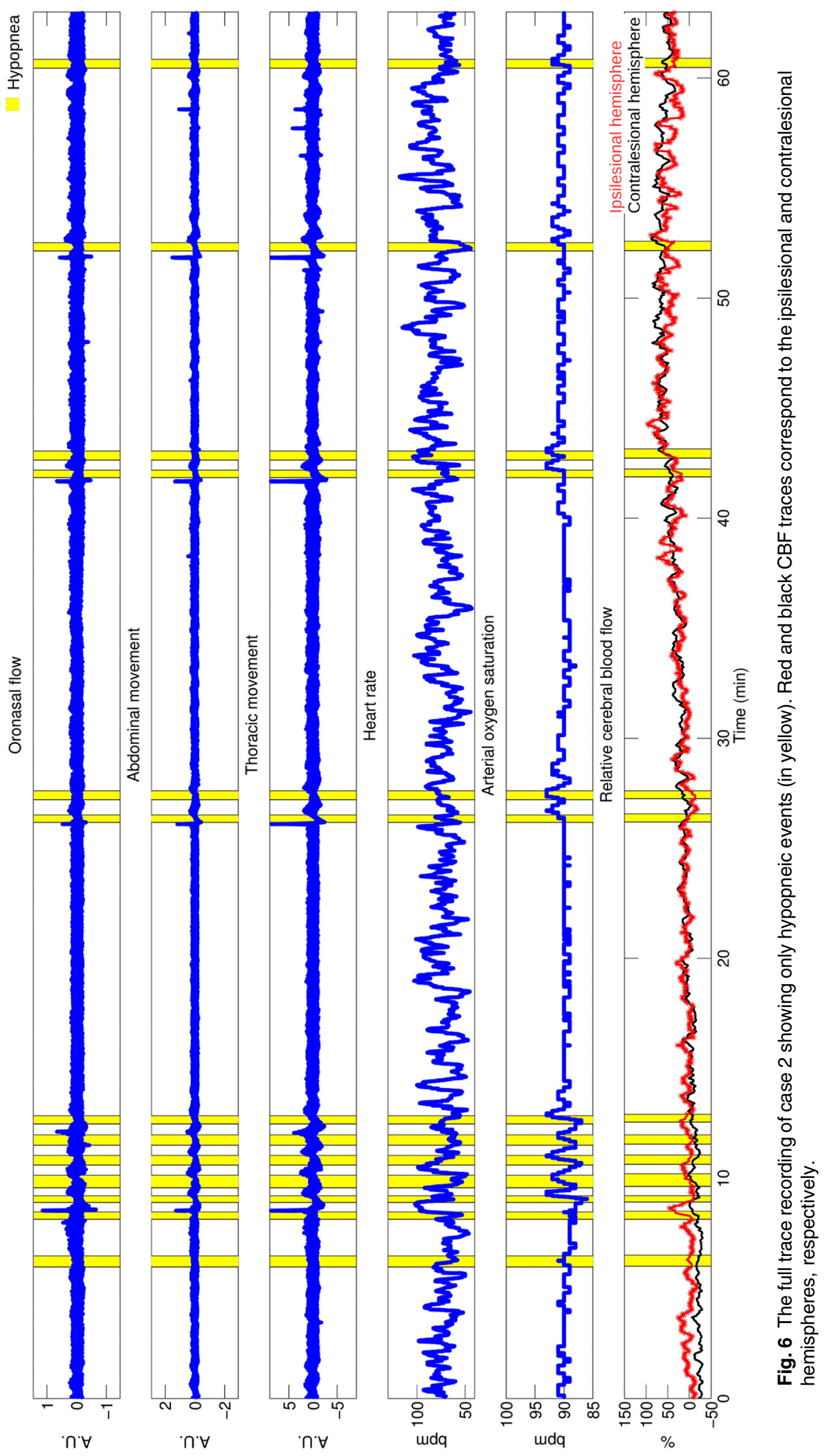




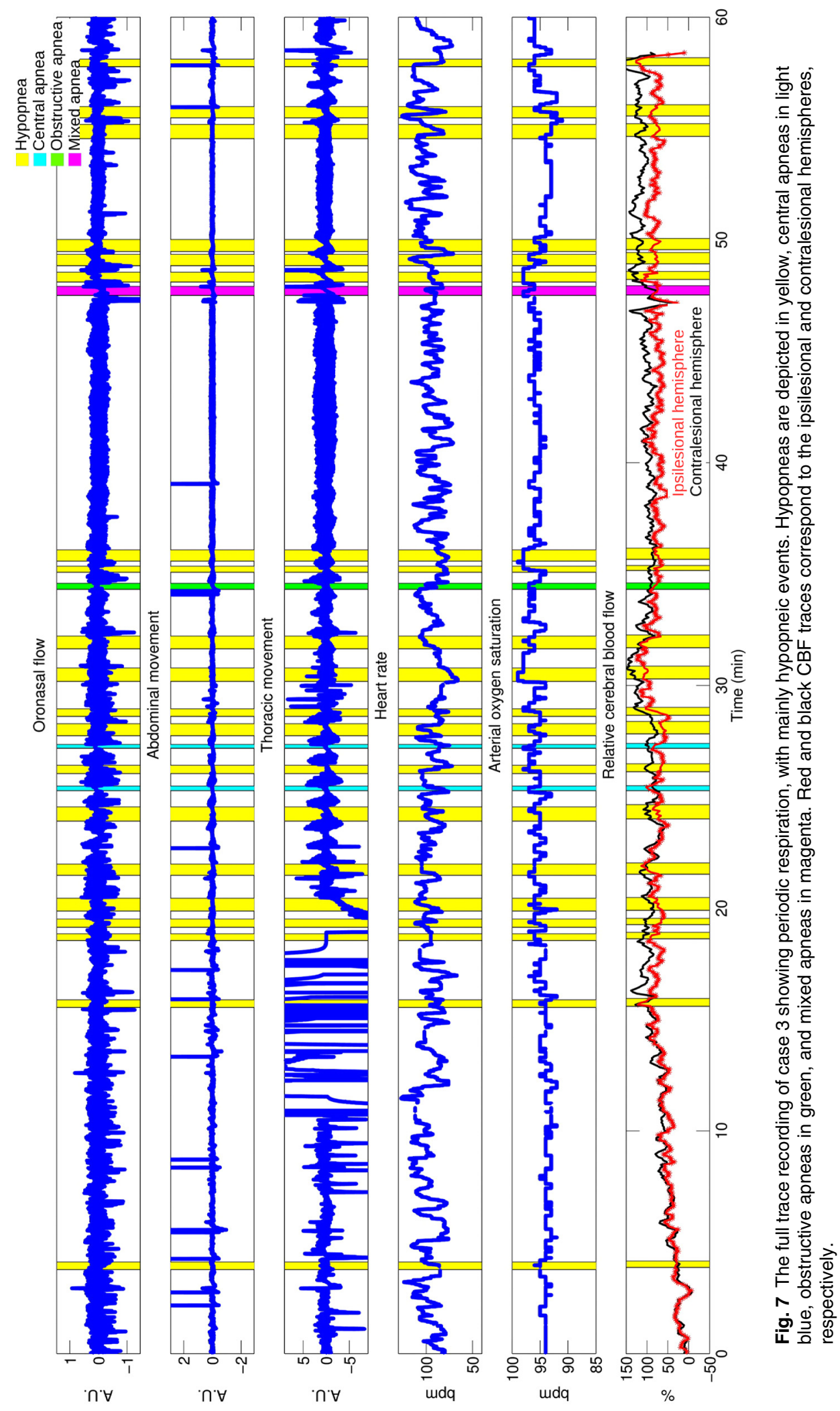




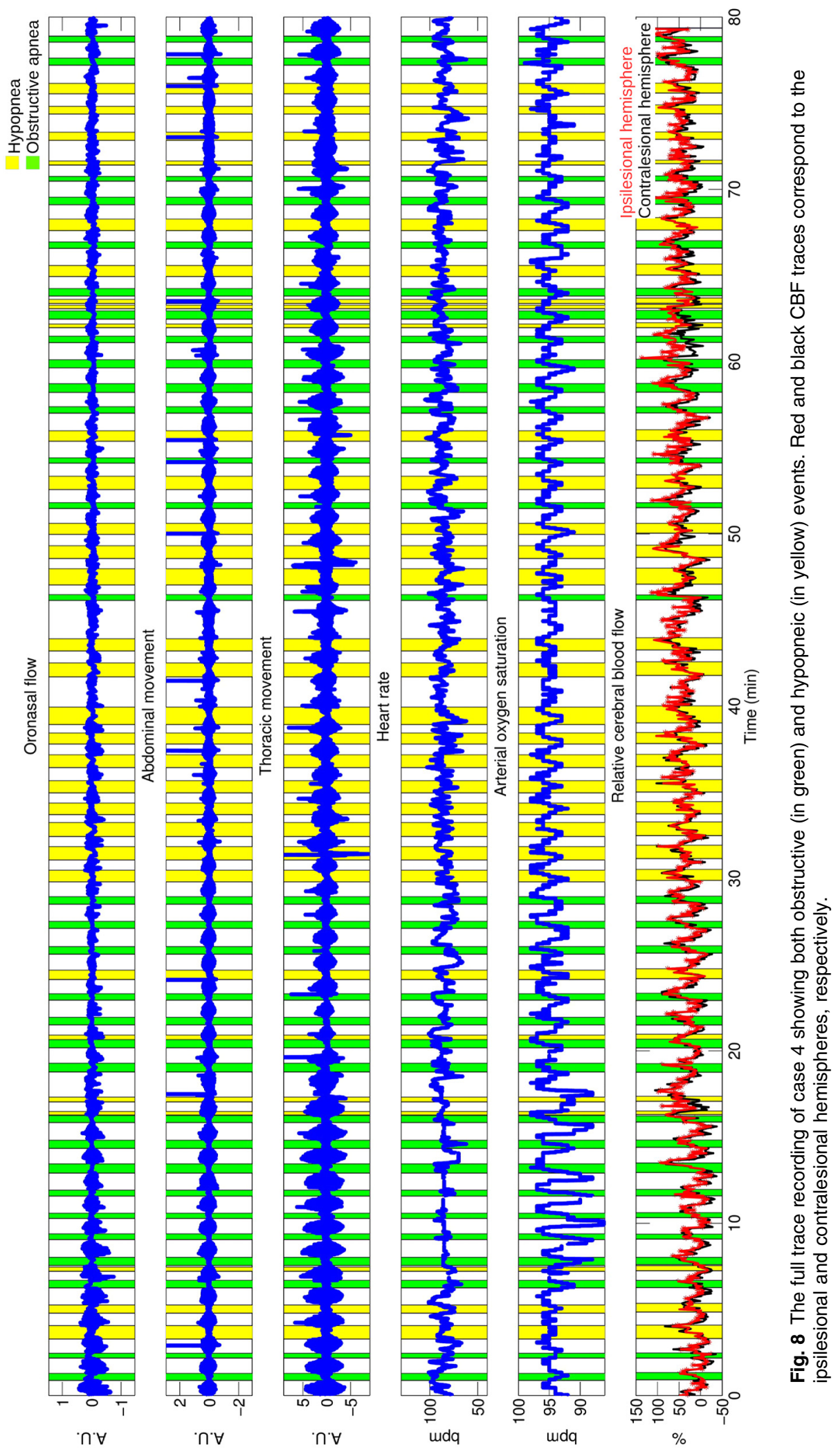




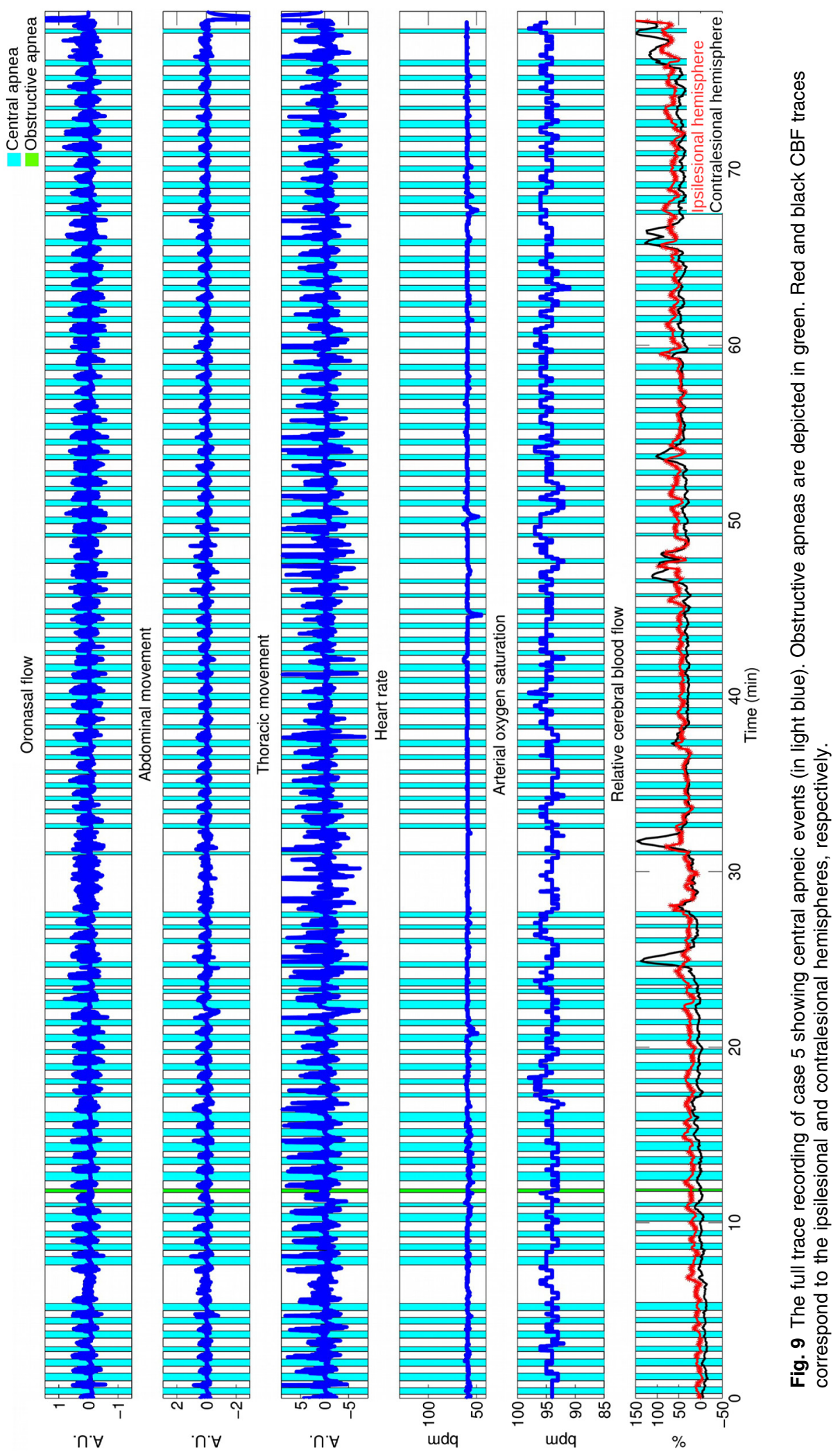




\section{Disclosures}

Herewith the following current or potential financial relationships are disclosed. ICFO has equity ownership in the spinoff company HemoPhotonics S.L. Potential financial conflicts of interest and objectivity of research have been monitored by ICFO Knowledge \& Technology Transfer Department. No financial conflicts of interest were identified.

\section{Acknowledgments}

The authors thank Dr. Arjun Yodh, Dr. John A. Detre, Dr. Janos Lückl, and Rosa Maria Miralda for their useful discussions. They acknowledge the support from Redes Temáticas de Investigación Cooperativa (RETICS-INVICTUS RD012/0014 and RETICS-INVICTUS PLUS RD16/0019/0010), Fundació CELLEX Barcelona, Ministerio de Economía y Competitividad/FEDER (PHOTODEMENTIA, DPI201564358-C2-1-R), Instituto de Salud Carlos III/FEDER (MEDPHOTAGE, DTS16/00087, FIS PI09/0557), the "Severo Ochoa" Programme for Centres of Excellence in R\&D (SEV-2015-0522), the Obra Social "la Caixa" Foundation (LlumMedBcn), Institució CERCA, AGAURGeneralitat (2017SGR-1555), LASERLAB-EUROPE IV (EUH2020 654148), "Fundació La Marató TV3" (201709.31), Marie Curie initial training network (OILTEBIA 317526), Societat Catalana de Pneumologia (SOCAP), and Sociedad Española de Neumología y Cirugía Torácica (SEPAR).

\section{References}

1. W. J. Powers et al., "2015 American Heart Association/American stroke association focused update of the 2013 guidelines for the early management of patients with acute ischemic stroke regarding endovascular treatment: a guideline for healthcare professionals from the American Heart Association/American Stroke Association," Stroke 46(10), 3020-3035 (2015).

2. I. Darien, International Classification of Sleep Disorders, 3rd ed. (ICSD-3), American Academy of Sleep Medicine (2014).

3. C. Bassetti and M. S. Aldrich, "Sleep apnea in acute cerebrovascular diseases: final report on 128 patients," Sleep 22(0161-8105 (Print)), 217-223 (1999).

4. A. Iranzo et al., "Prevalence and clinical importance of sleep apnea in the first night after cerebral infarction," Neurology 58(6), 911-916 (2002).

5. A. Nachtmann et al., "Cheyne-Stokes respiration in ischemic stroke," Neurology 45(4), 820-821 (1995).

6. C. Nopmaneejumruslers et al., "Cheyne-stokes respiration in stroke: relationship to hypocapnia and occult cardiac dysfunction," Am. J. Respir. Crit. Care Med. 171, 1048-1052 (2005).

7. M. Siccoli et al., "Central periodic breathing in 74 patients with acute ischemic stroke-neurogenic versus cardiogenic," J. Neuroimaging 255(11), 1687-1692 (2008).

8. D. L. Brown et al., "Brainstem infarction and sleep-disordered breathing in the BASIC Sleep Apnea Study," Sleep Med. 15(8), 887-891 (2014).

9. C. Bassetti, M. S. Aldrich, and D. Quint, "Sleep-disordered breathing in patients with acute supra- and infratentorial strokes: a prospective study of 39 patients," Stroke 28(9), 1765-1772 (1997).

10. D. C. Good et al., "Sleep-disordered breathing and poor functional outcome after stroke," Stroke 27(2), 252-259 (1996).

11. O. Parra et al., "Time course of sleep-related breathing disorders in firstever stroke or transient ischemic attack," Am. J. Respir. Crit. Care Med. 161(2 Pt 1), 375-380 (2000).

12. K. G. Johnson and D. C. Johnson, "Frequency of sleep apnea in stroke and TIA patients: a meta-analysis," J. Clin. Sleep Med. 6(2), 131-137 (2010).

13. A. M. Rowat, M. S. Dennis, and J. M. Wardlaw, "Central periodic breathing observed on hospital admission is associated with an adverse prognosis in conscious acute stroke patients," Cerebrovasc. Dis. 21(5-6), 340-347 (2006).

14. T. Cherkassky et al., "Sleep-related breathing disorders and rehabilitation outcome of stroke patients: a prospective study," Am. J. Phys. Med. Rehabil./Assoc. Acad. Physiatr. 82(6), 452-455 (2003).

15. M. Á. Martínez-García et al., "Continuous positive airway pressure treatment reduces mortality in patients with ischemic stroke and obstructive sleep apnea," Am. J. Respir. Crit. Care Med. 180(1), 36-41 (2009).

16. P. M. Turkington et al., "Effect of upper airway obstruction in acute stroke on functional outcome at 6 months," Thorax 59(5), 367-371 (2004).

17. O. C. Ioachimescu and N. A. Collop, "Sleep-disordered breathing," Neurol. Clin. 30, 1095-1136 (2012).

18. R. Aaslid et al., "Cerebral autoregulation dynamics in humans," Stroke 20(1), 45-52 (1989).

19. H. S. Markus, "Cerebral perfusion and stroke," J. Neurol. Neurosurg. Psychiatry 75(3), 353-361 (2004).

20. E. M. Bålfors and K. A. Franklin, "Impairment of cerebral perfusion during obstructive sleep apneas," Am. J. Respir. Crit. Care Med. 150, 1587-1591 (1994).

21. J. Klingelhofer et al., "Assessment of intracranial hemodynamics in sleep apnea syndrome," Stroke 23, 1427-1433 (1992).

22. G. Hajak et al., "Sleep apnea syndrome and cerebral hemodynamics," Chest 110(3), 670-679 (1996).

23. R. Alex et al., "Effect of apnea duration on apnea induced variations in cerebral blood flow velocity and arterial blood pressure," in 36th Annu. Int. Conf. Eng. Med. Biol. Soc. (EMBC), IEEE, 270-273 (2014).

24. M. Siebler and A. Nachtmann, "Cerebral hemodynamics in obstructive sleep apnea," Chest 103(4), 1118-1119 (1993).

25. M. J. Aries et al., "Near infrared spectroscopy for the detection of desaturations in vulnerable ischemic brain tissue," Stroke 43(4), 1134-1136 (2012).

26. F. Pizza et al., "Cerebral hemodynamic changes in stroke during sleepdisordered breathing," Stroke. 43, 1951-1953 (2012).

27. T. Durduran et al., "Diffuse optics for tissue monitoring and tomography," Rep. Prog. Phys. 73, 76701 (2010).

28. D. A. Boas, L. E. Campbell, and A. G. Yodh, "Scattering and imaging with diffusing temporal field correlations," Phys. Rev. Lett. 75(9), 18551858 (1995).

29. T. Durduran and A. G. Yodh, "Diffuse correlation spectroscopy for non-invasive, micro-vascular cerebral blood flow measurement," Neurolmage 85, 51-63 (2014).

30. R. C. Mesquita et al., "Direct measurement of tissue blood flow and metabolism with diffuse optics," Philos. Trans. A. Math. Phys. Eng. Sci. 369, 4390-4406 (2011).

31. C. Gregori-Pla et al., "Early microvascular cerebral blood flow response to head-of-bed elevation is related to outcome in acute ischemic stroke: a diffuse correlation spectroscopy study," in Int. Stroke Conf. 2017, American Heart Association, (Houston, USA) (2017).

32. T. Brott et al., "Measurements of acute cerebral infarction: a clinical examination scale," Stroke 20(7), 864-870 (1989).

33. P. A. Barber et al., "Validity and reliability of a quantitative computed tomography score in predicting outcome of hyperacute stroke before thrombolytic therapy," Lancet 355, 1670-1674 (2000).

34. J. C. Van Swieten et al., "Interobserver agreement for the assessment of handicap in stroke patients," Stroke 19(5), 604-607 (1988).

35. E. C. Jauch et al., "Guidelines for the early management of patients with acute ischemic stroke: a guideline for healthcare professionals from the American Heart Association/American Stroke Association," Stroke 44(3), 870-947 (2013).

36. P. Zirak et al., "Effects of acetazolamide on the micro- and macro-vascular cerebral hemodynamics: a diffuse optical and transcranial Doppler ultrasound study," Biomed. Opt. Express 1(5), 1443 (2010).

37. P. Zirak et al., "Microvascular versus macrovascular cerebral vasomotor reactivity in patients with severe internal carotid artery stenosis or occlusion," Acad. Radiol. 21, 168-174 (2014).

38. P. Zirak et al., "Transcranial diffuse optical monitoring of microvascular cerebral hemodynamics after thrombolysis in ischemic stroke," J. Biomed. Opt. 19(1), 018002 (2014).

39. R. Delgado-Mederos et al., "Transcranial diffuse optical assessment of the microvascular reperfusion after thrombolysis for acute ischemic stroke," Biomed. Opt. Express 9(3), 1262 (2018). 
40. R. B. Berry et al., "Rules for scoring respiratory events in sleep: Update of the 2007 AASM manual for the scoring of sleep and associated events," J. Clin. Sleep Med. 8(5), 597-619 (2012).

41. A. Roebuck et al., "A review of signals used in sleep analysis," Physiol. Meas. 35, R1-R57 (2014).

42. P. Zirak et al., "Characterization of the microvascular cerebral blood flow response to obstructive apneic events during night sleep," Neurophotonics 5, 1 (2018).

43. A. Leao, "Spreading depression of activity in cerebral cortex," $J$ Neurophysiol 7, 359-390 (1944).

44. J. P. Dreier et al., "Cortical spreading ischaemia is a novel process involved in ischaemic damage in patients with aneurysmal subarachnoid haemorrhage," Brain 132(7), 1866-1881 (2009).

45. A. J. Strong, J. A. Hartings, and J. P. Dreier, "Cortical spreading depression: an adverse but treatable factor in intensive care?" Curr. Opin. Crit. Care 13(2), 126-133 (2007).

46. H. Yanamoto et al., "Induced spreading depression activates persistent neurogenesis in the subventricular zone, generating cells with markers for divided and early committed neurons in the caudate putamen and cortex," Stroke 36(7), 1544-1550 (2005).

47. I. Aiba and J. L. Noebels, "Spreading depolarization in the brainstem mediates sudden cardiorespiratory arrest in mouse SUDEP models," Sci. Transl. Med. 7(282), 282ra46-282ra46 (2015).

48. A. D. Mcgown et al., "Measurement of changes in cytochrome oxidase redox state during obstructive sleep apnea using near-infrared spectroscopy," Sleep 26(6), 710-716 (2003).

49. A. Valipour et al., "Some factors affecting cerebral tissue saturation during obstructive sleep apnoea," Eur. Respir. J. 20(2), 444-450 (2002).

50. L. V. Pham and A. R. Schwartz, "The pathogenesis of obstructive sleep apnea," J. Thorac. Dis. 7(8), 1358-1372 (2015).

51. D. J. Durgan and R. M. Bryan, "Cerebrovascular consequences of obstructive sleep apnea," J. Am. Heart Assoc. 1, e000091 (2012).

52. O. B. Paulson, S. Strandgaard, and L. Edvinsson, "Cerebral autoregulation," Cerebrovasc. Brain Metab. Rev. 2, 161-192 (1990).

53. J. Wright et al., "Health effects of obstructive sleep apnoea and the effectiveness of continuous positive airways pressure: a systematic review of the research evidence," BMJ 314(7084), 851-860 (1997).

54. M. T. Naughton et al., "Effect of continuous positive airway pressure on central sleep apnea and nocturnal PCO2 in heart failure," Am. J. Respir. Crit. Care Med. 150(6 Pt 1), 1598-1604 (1994).

55. W. Randerath et al., "Definition, discrimination, diagnosis and treatment of central breathing disturbances during sleep," Eur. Respir. J. 49(1), 1600959 (2017).

56. C. M. Ryan et al., "Influence of continuous positive airway pressure on outcomes of rehabilitation in stroke patients with obstructive sleep apnea," Stroke 42(4), 1062-1067 (2011).

57. O. Parra et al., "Early treatment of obstructive apnoea and stroke outcome: a randomised controlled trial," Eur. Respir. J. Off. J. Eur. Soc. Clin. Respir. Physiol. 37(5), 1128-1136 (2011).

58. D. M. Bravata et al., "Continuous positive airway pressure: evaluation of a novel therapy for patients with acute ischemic stroke," Sleep 34(9), 1271-1277 (2011).

59. O. Sandberg et al., "Nasal continuous positive airway pressure in stroke patients with sleep apnoea: a randomized treatment study," Eur. Respir. J. 18, 630-634 (2001).

60. A. Gupta et al., "Role of positive airway pressure therapy for obstructive sleep apnea in patients with stroke: A randomized controlled trial," $J$. Clin. Sleep Med. 14(4), 511-521 (2018).

61. A.-K. Brill et al., "CPAP as treatment of sleep apnea after stroke," Neurology 90(14), e1222-e1230 (2018).

62. L. M. Tomfohr et al., "Continuous positive airway pressure for treatment of obstructive sleep apnea in stroke survivors: what do we really know?" Stroke. 43(11), 3118-3123 (2012).

63. M. E. Dyken and K. B. Im, "Sleep-disordered breathing and stroke," Sleep Med. Clin. 3(3), 361-376 (2008).

64. C.-Y. Hsu, "Sleep-disordered breathing after stroke: a randomised controlled trial of continuous positive airway pressure," J. Neurol. Neurosurg. Psychiatry 77(10), 1143-1149 (2006).

65. A. M. Rowat, J. M. Wardlaw, and M. S. Dennis, "Abnormal breathing patterns in stroke: relationship with location of acute stroke lesion and prior cerebrovascular disease," J. Neurol. Neurosurg. Psychiatry 78(3), 277-279 (2007).
66. D. M. Hermann et al., "Central periodic breathing during sleep in acute ischemic stroke," Stroke 38(3), 1082-1084 (2007).

67. T. Durduran et al., "Transcranial optical monitoring of cerebrovascular hemodynamics in acute stroke patients," Opt. Express 17(5), 38843902 (2009).

68. T. Przybylowski et al., "Mechanisms of the cerebrovascular response to apnoea in humans," J. Physiol. 548(1), 323-332 (2003).

69. H. Yaggi and V. Mohsenin, "Obstructive sleep apnoea and stroke," Lancet Neurol. 3(June), 333-342 (2004).

70. N. L. Chamberlin and L. Ling, "The effect of intermittent hypoxia on obstructive sleep apnea: beneficial or detrimental?" J. Appl. Physiol. 110, 9-10 (2011).

71. E. C. Fletcher, "Effect of episodic hypoxia on sympathetic activity and blood pressure," Respir. Physiol. 119, 189-197 (2000).

72. A. Torricelli et al., "Time domain functional NIRS imaging for human brain mapping," NeuroImage 85, 28-50 (2014).

73. J. Selb et al., "Sensitivity of near-infrared spectroscopy and diffuse correlation spectroscopy to brain hemodynamics: simulations and experimental findings during hypercapnia," Neurophotonics 1(1), 015005 (2014).

74. W. B. Baker et al., "Pressure modulation algorithm to separate cerebral hemodynamic signals from extracerebral artifacts," Neurophotonics 2(3), 035004 (2015).

Clara Gregori-Pla is a postdoctoral researcher in the group Medical Optics at ICFO-The Institute of Photonic Sciences, Spain. She received her master's degree in engineering physics in biomedical physics at the Royal Institute of Technology, Sweden, and her $\mathrm{PhD}$ in photonics at ICFO. Her research is focused on developing novel diffuse optical instrumentation, algorithms, and protocols and applying them for clinical studies.

Raquel Delgado-Mederos is a specialist in stroke neurology. She obtained her $\mathrm{PhD}$ in medicine at the Universitat Autònoma de Barcelona, Spain, in 2008. She works at the Stroke Unit of a comprehensive stroke center (Hospital de la Santa Creu i Sant Pau, Barcelona, Spain), in which she has professional experience as a clinician and as a researcher. Her research interests include acute stroke management, neuromonitoring, multimodal neuroimaging, neurosonology, and rehabilitation.

Gianluca Cotta is a medical doctor who obtained his training in pneumology at Hospital de la Santa Creu i Sant Pau in 2014. He studied for his master's degree in scientific research methodology at Universitat Autònoma de Barcelona. He is currently working in a hospital as a pneumologist.

Giacomo Giacalone obtained his degree in medicine, the specialty in neurology and his $\mathrm{PhD}$ in experimental neurology from the San Raffaele University in Milan, in 2008, 2014, and 2018, respectively. $\mathrm{He}$ works at the Stroke Unit of the Neurology Department of San Raffaele Hospital in Milan as a clinician and researcher. In 2015, he promoted the setup of a clinical research laboratory for the application of advanced optical device in ischemic stroke and other neurological diseases.

Federica Maruccia is a doctoral student in Neurotraumatology and Neurosurgery Research Unit (UNINN), Vall d'Hebron University Research Institute, Spain. She received her master's degree in rehabilitation therapy from the University of Pisa in 2013. The objective of her research is to evaluate the psychomotor development of babies and children affected by neurological diseases and to collaborate in the application of noninvasive technologies in order to better understand the pathological frame.

Stella Avtzi is a doctoral student in Medical Optics Group, at ICFO. She received her master's degree in optics and vision from the faculty of medicine at the University of Crete, Greece. Her research interests are mainly focused on developing new, noninvasive methods based on diffuse optics for their application in clinical studies.

Luís Prats-Sànchez is a neurologist in the Hospital de la Santa Creu i Sant Pau. He works in the Unit of Stroke Medicine since 2013. He has professional experience as a clinician and as a researcher in stroke medicine. In 2019, he will finish his doctoral thesis in medicine at the 
Universitat Autònoma de Barcelona. He is member of the Spanish and Catalan Societies of Neurology.

Alejandro Martínez-Domeño is a neurologist at the Hospital de la Santa Creu i Sant Pau. He works in the Unit of Stroke Medicine. $\mathrm{He}$ has professional experience as a clinician and as a researcher in stroke medicine.

Pol Camps-Renom is a specialist in stroke neurology. He was trained in the Calgary Stroke Program, Alberta, Canada. He is currently doing his PhD studies at the Universitat Autonoma de Barcelona, focusing on stroke prevention.

Joan Martí-Fàbregas is a specialist in stroke neurology (1988). He was trained in the Department of Neurology (Hospital de la Santa Creu i Sant Pau, 1988). He obtained his PhD in medicine at the Universitat Autònoma de Barcelona, in 1993. He is the director of the Stroke Unit at the Department of Neurology (Hospital de la
Santa Creu i Sant Pau). He has experience as a clinician and as a researcher. His research interests include acute stroke management, intracerebral hemorrhage, neurosonology, and stroke prevention.

Turgut Durduran was trained at the University of Pennsylvania, Philadelphia. In 2009, he moved to ICFO, where he leads the medical optics group. His research interests revolve around the use of diffuse light to noninvasively probe tissue function. The group develops new technologies and algorithms and routinely translates them to preclinical, clinical, and industrial applications.

Mercedes Mayos obtained her PhD in medicine from the Universitat Autònoma de Barcelona, where she is now an associate professor. She is a specialist in pneumology. She coordinates the Sleep Disorders Unit and leads the Pulmonary Function Unit in the Hospital de la Santa Creu i Sant Pau. Her research and work interests are related to respiratory sleep disorders and new study methods and clinical impact of obstructive sleep apnea-hypopnea syndrome. 